14. Egbert PR, Williams AS, Singh K, Dadzie P, Egbert TB. A prospective trial of intraoperative fluorouracil during trabeculectomy in a black population. Am J Ophthalmol 1993;116:612-6.

15. Dietze PJ, Feldman RM, Gross RL. Intraoperative application of 5FU during trabeculectomy. Ophthalmic Surg 1992;23:662-71.
16. Mora JS, Nguyen N, Iwach AG, et al. Trabeculectomy with intraoperative sponge 5-fluorouracil. Ophthalmology 1996;103:963-70.

17. Cordeiro MF, Constable PH, Alexander RA, Bhattacharya SS, Khaw PT. The effect of varying mitomycin-c treatment area in glaucoma filtration surgery in the rabbit. Invest Ophthalmol Vis Sci 1997;38:1639-46.

\title{
THE BRITISH OPHTHALMOLOGICAL SURVEILLANCE UNIT: THE STUDY OF UNCOMMON OPHTHALMIC DISORDERS MADE EASIER
}

A new national resource for ophthalmic research, the British Ophthalmological Surveillance Unit (BOSU) ${ }^{1}$ came into being in July 1997 with the support of the Iris Fund for the Prevention of Blindness and the Royal College of Ophthalmologists. At that time all senior ophthalmologists in the United Kingdom and Ireland were sent information about the aims, purposes and method of operation of the unit. Subsequently, a reporting card, listing the initial studies being facilitated by BOSU, has been sent each month to all associate specialists and consultants. In the early days of this new service it is timely to consider again why there is a need for a national system for ophthalmological surveillance and what the ophthalmological community will gain from the success of this new venture.

Medical surveillance of individual patients is intuitive to clinicians. It involves careful and systematic observation to detect early or evolving signs of disease and the institution of the appropriate interventions based on these observations. The practice of surveillance of whole populations for specific disorders may be less familiar to some ophthalmologists. This form of surveillance has been described as the 'continued watchfulness over the distribution and trends of incidence through systematic collection, consolidation and evaluation of morbidity reports and other relevant data'. ${ }^{2}$ The 'regular dissemination of the basic data and the interpretations to all who have contributed and to all others who need to know' is an intrinsic component of the process. ${ }^{2}$ Surveillance was initially used as a method for monitoring infectious diseases, an early example being William Farr's scrutiny of the 1848-9 cholera epidemic in England. ${ }^{3}$

Surveillance is no longer restricted to the study of infectious diseases. In Britain, the appropriateness and effectiveness of this method for the study of a variety of important but rare disorders is well recognised. It has already been implemented successfully, at a national level, in paediatrics ${ }^{4}$ and neurology, ${ }^{5}$ as well as in dermatology and orthopaedics. Population-based national surveillance effectively addresses the specific difficulties inherent in the study of uncommon diseases or events. It minimises the problem of the collection, in a reasonable time period, of the representative and sufficiently large number of cases that is necessary for unbiased and meaningful analysis in, and interpretation of, studies. In addition it is an effective means of early identification of important new or reemerging disorders that require prompt action, either to elucidate underlying causes, to review current treatment or to develop and implement new therapies.

The British Paediatric Surveillance Unit, ${ }^{4}$ the longest established of the speciality-specific systems in Britain, has achieved and maintained high levels of accurate reporting by paediatricians over the past decade. This may be related partly to the successful clinical paradigm of child health surveillance in which identifying and recording that a child is healthy is as important as noting any abnormalities. The equivalent in active epidemiological surveillance is that the effectiveness of the system depends as much on the confirmation by the majority of clinicians that no new cases of the disorder of interest have been seen as it does on actual notification of new cases by others. Whilst a similar example of health surveillance does not exist in ophthalmology, epidemiological surveillance is not untried. Recent examples of successful disorder-specific surveillance include that for Toxoplasma retinochoroiditis ${ }^{6}$ and for congenital cataract. $^{7}$ It is an undesirable burden for reporting clinicians and an inefficient and costly exercise for researchers, to continue to be involved in establishing surveillance systems for each study. A noted benefit of the other British surveillance systems is the reduction in separate mailings to clinicians about different studies. Furthermore, since all studies facilitated by these systems undergo extensive review before being supported, reporting clinicians can be more confident about the quality of the research programme to which they are asked to contribute. BOSU, drawing on the experiences of other systems, seeks to ensure that ophthalmologists benefit in the same ways.

Ophthalmological surveillance is most appropriate for the important uncommon disorders or events that would be difficult to identify in a representative, 
complete or timely way using other methods. Equally, the conditions to be studied must have clear importance to the ophthalmological community for at least one of the three following reasons: firstly, they are significant for public health or health policy; secondly, their study has important direct implications for clinical practice; and, thirdly, they offer the opportunity to study fundamental disease mechanisms. These requirements are illustrated by the range of studies currently being facilitated by BOSU. A study of Acanthamoeba keratitis and its relation to soft contact lens wear is a timely examination that has direct relevance to public health and clinical practice. Similarly, a study of the incidence and causes of loss of vision in the non-amblyopic eye amongst individuals with unilateral amblyopia addresses topical, unanswered questions important to the debate about childhood vision screening. By contrast, a study of sympathetic ophthalmia provides a new opportunity to characterise and clarify pathogenesis in a representative range of cases. Finally, a study of retinopathy of prematurity, to determine incidence and outcomes in a national cohort of affected children, will also gather information of importance to future provision of screening services for this disorder.

BOSU offers the ophthalmological community a new and effective medium for improving the study, thereby the understanding, and ultimately the management, of a variety of important but rare ophthalmic disorders. Everyone involved in clinical, basic scientific, or epidemiological research on such disorders is encouraged to consider this new service. The success of BOSU, and the studies it supports, depends on the continued interest and involvement of the reporting clinicians who, together with their patients, it ultimately serves. It is hoped that the ophthalmological community will continue to support this initiative to enhance the research base of ophthalmology.

J. R. is supported by the Medical Research Council.

Department of Epidemiology J. S. RAHI*

Institute of Child Health

London

Ipswich Hospital NHS Trust

C. EdELSTEN*

Ipswich

Suffolk

*On behalf of the Steering Committee of the British Ophthalmological Surveillance Unit.

\section{References}

1. Stanford M. Br J Ophthalmol 1997;81:932-3.

2. Langmuir AD. The surveillance of communicable diseases of national importance. N Engl J Med 1963; 268:182-91.

3. Farr W. Cholera epidemic, 1848-49. In: Humphreys NA, editor. Vital statistics: a memorial volume of selections from the reports and writings of William Farr. London: E Sanford, 1885:333-4.

4. British Paediatric Surveillance Unit. Lancet 1992;340: 845.

5. Cockerell OC, Gupta S, Catchpole M, Sander JW, Shorvan SD. The British Neurological Surveillance Unit: a nationwide scheme for the ascertainment of rare neurological disorders. Neuroepidemiology 1995;14:182-7.

6. Gilbert RE, Stanford MR, Jackson H, Holliman RE, Sanders MD. The incidence of acute symptomatic Toxoplasma retinochoroiditis in south London according to country of birth. BMJ 1995;310:1037-40.

7. The British Congenital Cataract Study. British Paediatric Surveillance Unit Annual Report 1996. London: Royal College of Paediatrics and Child Health, 1997.

\section{HOW SHOULD ACTIVE TOXOPLASMIC RETINOCHOROIDITIS BE TREATED?}

Despite a plethora of publications concerning Toxoplasma and toxoplasmosis,${ }^{1}$ clinical diagnosis of the ocular disease remains subjective, and treatment largely empirical. This is due, at least in part, to a lack of consistent data pertaining to the host-parasite relationship within the human uveo-retinal tract, and particularly whether, in vivo, Toxoplasma is actually responsible for initiation and perpetuation of the ocular disease. Such knowledge is necessary if there is to be a rational approach to the medical therapy of ocular toxoplasmosis. ${ }^{2}$

The Toxoplasma tissue cyst may be the major contributor to the ongoing pathogenetic process. Electron microscopy of animal ocular tissue with optimal fixation has revealed that intact tissue cysts in the retina are invariably located within viable cells in the ganglion cell and/or inner nuclear layers. ${ }^{3}$ Demise of the host cell liberates viable toxoplasms and other Toxoplasma antigens into the surrounding host tissue, to elicit a localised inflammatory event and, perhaps, permitting parasitisation of adjacent cells. Activated macrophages and other immune cells process the Toxoplasma antigen, ${ }^{4}$ thus suppressing the infection. Medical intervention is usually necessary, however, to reduce progression of inflammation, especially if the lesion is considered clinically to be sight-threatening. ${ }^{5}$

Proposed chemotherapeutic strategies for toxoplasmic retinopathy, ${ }^{6}$ including cases associated with pregnancy, neonates and the severely immunocom- 